\title{
Expression of LOC285758, a potential long non-coding biomarker, is methylation- dependent and correlates with glioma malignancy grade
}

\author{
Alenka Matjasic ${ }^{1}$, Mara Popovic², Bostjan Matos³ and Damjan Glavac ${ }^{1}$ \\ ${ }^{1}$ Department of Molecular Genetics, Institute of Pathology, Faculty of Medicine, University of Ljubljana, Slovenia \\ ${ }^{2}$ Institute of Pathology, Faculty of Medicine, University of Ljubljana, Slovenia \\ ${ }^{3}$ Department of Neurosurgery, University Medical Center, Ljubljana, Slovenia
}

Radiol Oncol 2017; 51(3):331-341.

Received 24 October 2016

Accepted 22 November 2016

Correspondence to: Prof. Dr. Damjan Glavač, Department of Molecular Genetics, Institute of Pathology, Faculty of Medicine, University of Ljubljana, Korytkova 2, SI-1000 Ljubljana, Slovenia. E-mail: damjan.glavac@mf.uni-lj.si, Tel.: +386-1-543-7180

Disclosure: No potential conflicts of interest were disclosed.

Background. Identifying the early genetic drivers can help diagnose glioma tumours in their early stages, before becoming malignant. However, there is emerging evidence that disturbance of epigenetic mechanisms also contributes to cell's malignant transformation and cancer progression. Long non-coding RNAs are one of key epigenetic modulators of signalling pathways, since gene expression regulation is one of their canonical mechanisms. The aim of our study was to search new gliomagenesis-specific candidate IncRNAs involved in epigenetic regulation.

Patients and methods. We used a microarray approach to detect expression profiles of epigenetically involved IncRNAs on a set of 12 glioma samples, and selected LOC285758 for further qPCR expression validation on $157 \mathrm{glioma}$ samples of different subtypes. To establish if change in expression is a consequence of epigenetic alterations we determined methylation status of IncRNA's promoter using MS-HRM. Additionally, we used the MLPA analysis for determining the status of known glioma biomarkers and used them for association analyses.

Results. In all glioma subtypes levels of LOC285758 were significantly higher in comparison to normal brain reference RNA, and expression was inversely associated with promoter methylation. Expression substantially differs between astrocytoma and oligodendroglioma, and is elevated in higher WHO grades, which also showed loss of methylation. Conclusions. Our study revealed that IncRNA LOC285758 changed expression in glioma is methylation-dependent and methylation correlates with WHO malignancy grade. Methylation is also distinctive between astrocytoma I-III and other glioma subtypes and may thus serve as an additional biomarker in glioma diagnosis.

Key words: glioma; IncRNA; LOC285758; over-expression; epigenetics; DNA methylation; MS-HRM; MLPA; IDHI; 1p/19q

\section{Introduction}

Glioma are the commonest primary brain tumours in adults and also the most aggressive, with overall poor survival. ${ }^{1,2}$ Clinically, they are extremely heterogeneous primary brain tumours that are presenting a great challenge in clinical oncology. This heterogeneity is reflected in the lack of knowledge about the exact mechanisms of tumour formation and progression, subsequently leading to less ac- curate classification and choosing the appropriate treatment. It is why numerous researchers have focused to find new genetic factors and molecular mechanisms involved in gliomagenesis, which may also contribute to developing new therapeutic approaches and improving the prognosis for glioma patients.

LncRNAs are widely expressed in mammalian nervous system ${ }^{3}$ and several were identified to be specifically linked with neuro-oncological 
TABLE 1. Patients' demographics and glioma histopathological classification

\begin{tabular}{|c|c|c|}
\hline \multicolumn{3}{|l|}{ Patients demographic } \\
\hline Number of patients & \multicolumn{2}{|l|}{157} \\
\hline Gender (female/male) & \multicolumn{2}{|l|}{$67 / 90(1: 1.34)$} \\
\hline Mean age at diagnosis (years) & \multicolumn{2}{|l|}{$43.8(S D \pm 18,89)$} \\
\hline$\#<45$ years & \multicolumn{2}{|l|}{86} \\
\hline$\#>45$ years & \multicolumn{2}{|l|}{71} \\
\hline Glioma classification & Glioma subtype & WHO grade \\
\hline \multirow[t]{6}{*}{ Astrocytoma (AC) } & 15 pilocytic & WHO I \\
\hline & 9 diffuse & WHO ॥ \\
\hline & 11 diffuse with signs of anaplasia & WHO II-III \\
\hline & 9 anaplastic & WHO III \\
\hline & 23 secondary GBM & WHO IV \\
\hline & 31 primary GBM & WHO IV \\
\hline \multirow[t]{3}{*}{ Oligodendroglioma (ODG) } & 4 diffuse & WHO ॥ \\
\hline & 5 diffuse with signs of anaplasia & WHO $\|-I I I$ \\
\hline & 28 anaplastic & WHO III \\
\hline \multirow[t]{3}{*}{ Oligoastrocytoma (OAC) } & 2 diffuse & WHO ॥ \\
\hline & 3 diffuse with signs of anaplasia & WHO $\|-I I I$ \\
\hline & 17 anaplastic & WHO III \\
\hline
\end{tabular}

disorders ${ }^{4,5}$, and as such they could help explain the mechanisms of glioma malignant transformation. ${ }^{6,7}$ LncRNAs are a class of non-coding RNAs that share many features with protein-coding RNAs (mRNAs), but lack the open reading frame, have lower sequence conservation and lower level of expression. ${ }^{8,9}$ However, they are more cell- and tissue-type specific than mRNAs. ${ }^{10}$ For many of them functional analyses showed to have key roles in numerous fundamental biological processes, such as cell cycle regulation, epigenetic regulation, imprinting, cell differentiation and apoptosis, and diseases, including tumorigenesis. ${ }^{11-17}$ The involvement of lncRNAs in disease processes as one of the most important factors controlling gene expression creates an urgency to understand the mechanisms by which these RNAs seek their targets and impact signalling pathways ${ }^{16}$, and how much their disturbance might contribute to disease development.

Gene expression regulation by lncRNAs appears to be mediated largely through epigenetic mechanisms, which play a crucial role in regulating gene expression and are closely associated with disease onset. A number of studies show that as much as $20-30 \%$ of lncRNAs have been able to physically interact with specific epigenetic enzymes and driving them to specific genomic loci. ${ }^{18} \mathrm{By}$ binding to the chromatin-modifying proteins, such as PRC2,
G9a, hnRNPK, and SWI/SNF, they modulate the chromatin states and thus impact gene expression of cell cycle, cell differentiation, apoptosis, DNA repair, and cell adhesion., ${ }^{3,17,19}$ Moreover, they can directly modulate the transcription of proximallylocated genes by interacting with promoters and transcription factors. ${ }^{3}$

In glioma, epigenetic alterations and mechanisms, including methylation of gene promoters, histone modifications and chromatin modifiers are relatively unexplored, although DNA methylation of O6-methylguanin-DNA methyltransferase (MGMT) expression was associated with the prognosis of glioma patients. ${ }^{20,21}$ DNA methylation is one of the primary epigenetic mechanisms in regulating gene expression, and disturbance of this process may cause various diseases, including cancer. It is also the most common epigenetic modification found in tumour cells. ${ }^{22}$ Moreover, DNA methylation is an important regulator of expression of not only the mRNAs but also of the lncRNAs, and it seems that DNA demethylation of silent lncRNAs results in their activation. ${ }^{23}$ Methylation patterns differ among various tissue-types and cell-types ${ }^{24}$, which means the cell-/tissue-specific methylation status, similarly to expression pattern, could be a useful biomarker.

In this study, we performed an expressional profiling of lncRNAs potentially involved in epigenetic levels of regulation in glioma samples of different histological subtypes compared to normal brain reference RNA. We used the microarray approach (LncPath ${ }^{\mathrm{TM}}$ Human Epigenetic Pathway) to search for novel lncRNA biomarkers potentially involved in glioma development. To validate microarray profiling results we used the qPCR method on a set of 125 glioma samples of different subtype and malignancy grade. In addition, we wanted to investigate if the change in gene's expression is due to changed methylation pattern of its promoter, and if there is any association with the hallmark markers, such as IDH1/2 and TP53 mutation, copy number variation of genes $C D K N 2 A$ and $C D K N 2 B$, and $1 \mathrm{p} / 19 \mathrm{q}$ co-deletion.

\section{Patients and methods}

\section{Patients}

Hundred and fifty-seven tumours that were surgically removed from patients between the years 2007 and 2015 were chosen for the study. Immediately after surgical biopsy tumours were stabilized in RNAlater (Applied Biosystems, USA), incubated at 
$4^{\circ} \mathrm{C}$ for at least 24 hours and subsequently stored at $-20^{\circ} \mathrm{C}$ until needed. All tumours were evaluated by neuro-pathologist in order to assess glioma subtype and tumour grade (see Table 1), according to the WHO 2007 guidelines. ${ }^{25}$ We collected patient's demographic data and results of immunohistochemical analyses, if they were performed, from our institutional database. The tumour biopsies used in the study belonged to 67 female and 90 male patients (mean age at diagnosis: 43.8 years). For reference RNA we used commercially available FirstChoice Human Brain Reference Total RNA (Cat.no. 6050, Ambion; Invitrogen, USA) (further referred to as brain reference RNA) obtained from the healthy brain tissue of 23 individuals without any signs of neurodegeneration. The study was approved by the National Medical Ethics Committee of the Republic of Slovenia (115/5/14). As DNA control samples we used nine samples of freshly frozen brain tissue that were collected at the autopsy of five patients ( 5 cerebrum and 4 cerebellum tissue samples). Samples were evaluated as a normal brain tissue, without any visible signs of neurodegeneration.

\section{Nucleic acid extraction}

For extraction and purification of nucleic acids we used the AllPrep DNA/RNA/miRNA Universal Kit (Qiagen, Germany). Total DNA and RNA were simultaneously extracted from the same piece of tissue (up to $20 \mathrm{mg}$ ) that was homogenized with TissueLyser LT (Qiagen, Germany) for 5 min at 50 $\mathrm{Hz}$, and further processed according to manufacturer's instructions. The yield of both RNA and DNA was measured spectrophotometrically using the NanoDrop ND-1000 (Thermo Scientific, USA). The quality and fragmentation of the extracted RNA (RNA integrity) was determined by agarose gel electrophoresis on Bioanalyzer 2100 (Agilent Technologies, USA), using the RNA 6000 Nano kit (Agilent Technologies, USA) according to manufacturer's instructions. Only the samples with appropriate yield and quality were considered for further analyses; thus, not all samples were used for all analyses performed.

\section{Expressional profiling of epigenetically involved IncRNA}

We performed expressional profiling on 12 RNA samples of different glioma subtype and a brain reference RNA. Samples were chosen from a sample set of our previous glioma study (unpublished study), and selected upon histopathologically determined glioma subtypes so that each of the four subgroups, i.e. astrocytoma (WHO grade II or III), primary GBM, secondary GBM and oligodendroglioma (WHO grade III), included 3 samples of sufficient RNA concentration and high quality. Samples were sent to ArrayStar Inc, USA, where sample preparation and microarray hybridization were performed, based on the manufacturer's standard protocols (Arraystar Inc.). One $\mu \mathrm{g}$ of RNA for individual sample was hybridized to the LncPath Human Epigenetic Pathway microarray (6x7K). Scanned slide images were processed by GenePix Pro 6.0 software (Axon) for raw data extraction, which were further normalized and processed using the $\mathrm{R}$ language. After filtering, only the probes for which at least 2 out of 13 samples had raw intensity above 32 were retained for further differential analyses. LncRNAs that showed significant change in expression between tumour group and brain reference RNA were identified through volcano plot filtering. After identifying groups' candidate genes, each group was compared to others. The »fold change« cut-off value for IncRNA to be considered as differentially expressed was set to $<-1.5$ (expression is decreased) and $>1.5$ (expression is increased), and p-value cutoff was set at 0.05 .

\section{Quantitative real-time PCR (qPCR) validation of LOC285758 expression}

LOC285758 expression levels were validated with the quantitative real-time PCR method, based on the intercalating dye (SYBR Green) technology. Only the samples with total RNA concentration higher than $50 \mathrm{ng} / \mu \mathrm{L}$ and RIN above 5.5 were used for qPCR analysis. The first strand cDNA was generated with One Taq RT-PCR kit according to manufacturer's instructions (New England Biolabs, UK) and using random primer mix. The reverse transcription reaction was prepared in a $10 \mu \mathrm{L}$ reaction mixture with $300 \mathrm{ng}$ of total input RNA. cDNA was properly diluted and amplified in a $10 \mu \mathrm{L}$ reaction volume, using the SYBR Select Master Mix (Thermo Fisher Scientific, USA). We used GAPDH and U6 as reference genes (endogenous controls) and brain reference RNA (mentioned in section 2.1) as the control RNA. All qPCR reactions were performed in duplicate using the Rotor Gene-Q system (Qiagen, Germany) following the primer manufacturer's standard cycling protocol for pre-designed primers for LOC285758 (PrimeTime qPCR Assay primer, IDT - Integrated 
TABLE 2. Primers used for validation of LOC285758 expression profiling results, reference genes and determining methylation status of IncRNA's promoter

\begin{tabular}{|c|c|c|c|}
\hline \multicolumn{4}{|c|}{ Quantitative real-time PCR } \\
\hline Gene & Assay ID & Amplicon length (bp) & Annealing temperature $\left({ }^{\circ} \mathrm{C}\right)$ \\
\hline LOC285758 & Hs.PT.58.26012748 & 129 & 60 \\
\hline GAPDH & QT00079247 & 95 & 55 \\
\hline Gene & Primer sequence $\left(5^{\prime}-3^{\prime}\right)$ & Amplicon length (bp) & Annealing temperature $\left({ }^{\circ} \mathrm{C}\right)$ \\
\hline \multirow[t]{2}{*}{ U6 } & CTCGCTTCGGCAGCACA & 94 & 60 \\
\hline & AACGCTTCACGAATTTGCGT & & \\
\hline \multicolumn{4}{|c|}{ Methylation sensitive HRM } \\
\hline Gene & Oligonucleotide sequence $\left(5^{\prime}-3^{\prime}\right)$ & Amplicon length (bp) & Annealing temperature $\left({ }^{\circ} \mathrm{C}\right)$ \\
\hline LOC285758 F & TTGTTTTTTGAAAGTTTTTTGA & 118 & 55 \\
\hline LOC285758 R & AAACACAAAAAACCTAACAAAAA & & \\
\hline
\end{tabular}

DNA Technology, USA) and GAPDH (QuantiTect qPCR Primer Assay, Qiagen, Germany). The cycling protocol for designed $U 6$ primers was similar to that of Qiagen with optimized $\mathrm{T}_{\mathrm{A}}$ (see Table 2). The signal was collected on the Green channel at the end point of every cycle, and following amplification melt curve analysis was performed to verify specificity of qPCR amplicon.

Relative quantification levels of the target gene were calculated following the $\Delta \Delta C_{T}$ method; $\Delta \Delta C_{T}$ represents the difference between the quantity of target transcript in brain reference RNA (control RNA) $\left(\Delta \mathrm{C}_{\mathrm{T}}\right.$ control) and in tumour $\left(\Delta \mathrm{C}_{\mathrm{T}}\right.$ sample $)$, after each sample and control were normalized to geometric mean expression of reference genes (GAPDH and $U 6) .{ }^{26} \mathrm{~A}$ positive $\triangle \Delta \mathrm{C}_{\mathrm{T}}$ value in our calculations means higher expression level in tumour samples.

\section{Methylation-sensitive HRM}

For determining lncRNA's promoter methylation status we used the methylation sensitive high resolution melt (MS-HRM) analysis. Primers for amplifying LOC285758 promoter's target region were designed with a freely available software tool Methyl Primer Express software v1.0 (Applied Biosystems, USA) in such a manner that they amplify both methylated and unmethylated DNA. Primers were designed to target $\mathrm{CpG}$ specific region at 5'UTR end (flanking sequence/exon1). Prior to MS-HRM analysis, $500 \mathrm{ng}$ of input amount of sample DNA were bisulphite converted (bsDNA) using innuCONVERT Bisulfite Basic Kit (Analytik Jena AG,
Germany) according to manufacturer's protocol. We created two DNA control pools by mixing 5 control samples of cerebrum and 4 control samples of cerebellum, treated them with bisulphite and used them for comparing the methylation status between normal brain tissue and tumour samples. For fully methylated/unmethylated (positive/ negative) controls we used commercially available EpiTect Control DNA (Qiagen, Germany). All MS-HRM reactions were prepared with EpiTect HRM PCR Kit (Qiagen, Germany) in a $10 \mu \mathrm{L}$ reaction mixture by manufacturer's recommendations and adjustments according to primer optimization analysis $(1.5 \mu \mathrm{L}$ of each primer, $1 \mu \mathrm{L}$ bsDNA $(10 \mathrm{ng} / \mu \mathrm{L}))$. We included the negative and positive control, both control pools and no-template control in each MS-HRM experiment, which were carried out on Rotor Gene Q (Qiagen, Germany). Amplification conditions were set based on manufacturer's recommendations as follows: 5 minutes of initial denaturation at $95^{\circ} \mathrm{C}, 45$ cycles: denaturation at $95^{\circ} \mathrm{C}$ for 10 seconds, annealing at optimized temperature $\left(T_{A}\right.$ in Table 2$)$ for 30 seconds, and elongation at $72^{\circ} \mathrm{C}$ for 20 seconds. After amplification, the HRM analysis was conducted by increasing the temperature from $65^{\circ} \mathrm{C}$ to $95^{\circ} \mathrm{C}$ by $0.1^{\circ} \mathrm{C}$ per 2 seconds. Fluorescence signal was collected from the green channel in elongation step and from the HRM channel during HRM analysis. For analysing MS-HRM results, we used the Rotor-Gene Q Series Software 2.3.1 (Qiagen, Germany). For determining methylation status of individual sample, samples HRM melting plots were normalized and further compared to controls. 


\section{Multiplex ligation-dependent probe amplification}

We used the P088-C1 Oligodendroglioma SALSA MLPA probe mix (MRC-Holland, the Netherlands) to detect loss of chromosome arms $1 p$ and $19 q$, copy number variations in $C D K N 2 A$ and $C D K N 2 B$ genes, and to determine the status of the most common mutations of IDH1 (R132H and R132C) and IDH2 (R172K and R172M) genes in different glioma subtypes. The results of MLPA analysis have been further considered as the criteria for sample sub-classification for additional comparison, and determining possible differences in gene expression and promoter methylation. The MLPA experiment was prepared according to manufacturer's protocol and recommendations with $100 \mathrm{ng}$ of input DNA amount. Capillary gel electrophoresis was performed using ABI Prism 310 Genetic Analyser (Applied Biosystems, USA), and we used Coffalyser software (MRC-Holland, the Netherlands) for fragment analysis.

\section{Data analysis}

All statistical tests were performed using IBM SPSS Statistics 20. software (IBM Corporation, New York, USA). We used the one-way ANOVA analysis for comparing differences in expression between all glioma subtypes, and Mann-Whitney 2-independent test to cross test differences between two subtypes. Pearson's correlation coefficient was used to establish association of expression with promoter's methylation status, status of known biomarkers, and glioma subtype. Differences were considered statistically significant when they reached or were below $\mathrm{p} \leq 0.05$.

\section{Results}

\section{Expression profiling of epigenetically involved IncRNAs}

Expression profiling of 12 glioma tumour samples of four different subtypes was performed with an ArrayStar microarray technology. Differential analysis of tumour samples compared to brain reference RNA, with absolute fold change (FC) cut-off value at 1.5 , showed $351 \mathrm{lncRNAs}$ with altered expression levels in at least one subtype (Figure 1A,B). Among these, 60 lncRNAs were differentially expressed in all four subtypes (Figure 1B). A more stringent analysis with absolute FC cut-off set at 2 showed 187 lncRNAs with altered expression
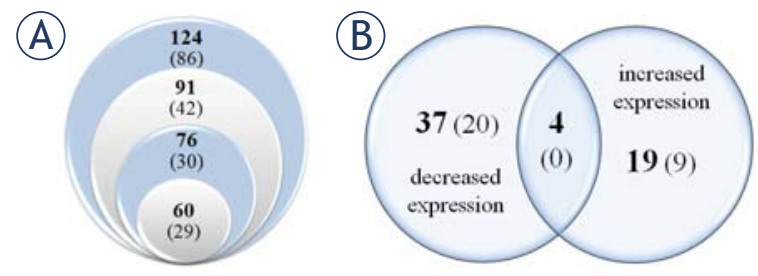

FIGURE 1. Venn's diagram of IncRNAs that were significantly differentially expressed using microarray screening of IncRNAs involved in epigenetic mechanisms and/or pathways. (A) Number of IncRNAs in regard to the number of subtypes in which they were found differentially expressed (the number of subtypes rises from the outer circle (one subtype) towards the inner one (four subtypes)). (B) The number of IncRNAs found differentially expressed in all four analysed subtypes (using two levels of stringency - absolute fold change cut-off value of 1.5 and (2)).

in at least one subtype and only 29 lncRNAs in all four subtypes (Figure 1A,B; numbers of lncRNAs matching this criteria are in parentheses). In Table 3 are listed top 10 over-expressed and top 10 under-expressed lncRNAs in each glioma subtype.

With the purpose to identify new potential biomarker candidates we searched through public databases, such as PubMed (https://www.ncbi.nlm. nih.gov/pubmed/), Ensembl (http://www.ensembl. org/index.html), HGNC (http://www.genenames. org/), and lncRNAdb (http://lncrnadb.com/), for information about gene's location in the genome, its known function or involvement in cellular pathways, and previous mention in the literature. Among the most differentially changed was lncRNA LOC285758, which showed increased expression in all four glioma subtypes, but lower expression in GBMs in comparison to astrocytoma and oligodendroglioma (Table 3). Also, it was significantly changed between the two GBM subtypes.

\section{Validation of LOC285758 expression}

To validate the microarray expression results of LOC285758 we used the quantitative real-time PCR method on a subset of 125 samples that met both the concentration and quality criteria. Expression levels were significantly increased in 105/125 samples (84\%) compared to brain reference RNA (Figure 2A), and all glioma subtypes showed positive $\Delta \Delta C_{T}$ average values (Figure $2 B$ ). Data obtained by qPCR validation analysis were in concordance with results of microarray profiling that showed increased levels in all four subtypes (Figure 2B). However, the expression in GBMs did not significantly differ, as observed on microar- 
TABLE 3. Top 10 IncRNAs that showed significantly increased/decreased expression in four glioma subtypes, using the LncPath Human Epigenetic Pathway microarray (ArrayStar, USA)

\begin{tabular}{|c|c|c|c|c|c|c|c|}
\hline \multicolumn{2}{|c|}{ Astrocytoma II+III* } & \multicolumn{2}{|c|}{ Secondary GBM } & \multicolumn{2}{|c|}{ Primary GBM } & \multicolumn{2}{|c|}{ Oligodendroglioma } \\
\hline $\mathrm{FC}$ (abs) & Gene Name & $\mathrm{FC}(\mathrm{abs})$ & Gene Name & $\mathrm{FC}$ (abs) & Gene Name & FC(abs) & Gene Name \\
\hline \multicolumn{8}{|c|}{ TOP 10 OVER-EXPRESSED } \\
\hline 9.775 & RP1 1-434O22.1 & 9.840 & APOC2 & 11.343 & AK024556 & 10.085 & RP6-201G10.2 \\
\hline 7.863 & LOC285758 & 9.105 & AK024556 & 9.761 & FJ209302 & 7.233 & LOC285758 \\
\hline 6.203 & LOC 100129034 & 7.971 & LOC 100129034 & 9.402 & AK055628 & 6.241 & GAS5 \\
\hline 5.247 & RP11-264F23.3 & 7.578 & AK055628 & 9.267 & $\mathrm{H} 19$ & 5.454 & RP11-264F23.3 \\
\hline 5.211 & RP6-201G10.2 & 4.509 & RP11-145M9.3 & 7.012 & RP11-434O22.1 & 5.360 & LOC 100216546 \\
\hline 5.107 & APOC2 & 4.243 & RPI1-73E17.2 & 6.720 & APOC2 & 5.043 & SNRPE \\
\hline 4.374 & RPI1-770J1.3 & 3.657 & KB-1836B5.1 & 5.527 & LOC285758 & 4.991 & AK024556 \\
\hline 4.211 & HOXAII-AS & 3.394 & H19 & 4.851 & LOC 100216546 & 4.930 & AC009506.1 \\
\hline 3.861 & RP3-405J24.1 & 2.878 & BANCR & 4.770 & LOC 100129034 & 4.351 & RPI 1-73E 17.2 \\
\hline 3.795 & AK055628 & 2.695 & $A B 447886$ & 4.525 & HOXA11-AS & 3.846 & LOC286059 \\
\hline \multicolumn{8}{|c|}{ TOP 10 UNDER-EXPRESSED } \\
\hline 9.638 & RP11-678P16.1 & 24.555 & MEG3 & 22.494 & MEG3 & 43.328 & RP11-678P16.1 \\
\hline 7.026 & XLOC_013368 & 11.341 & AK054921 & 16.532 & AK054921 & 23.879 & FABP5P3 \\
\hline 6.797 & AK054921 & 8.050 & AF520792 & 11.157 & RP11-678P16.1 & 18.840 & DGCR5 \\
\hline 6.148 & MEG3 & 6.845 & DGCR5 & 8.208 & DGCR5 & 8.073 & MEG3 \\
\hline 6.003 & RPI1-18F14.2 & 6.623 & XLOC_013368 & 8.207 & XLOC_013368 & 7.092 & AK054921 \\
\hline 5.820 & HARIA & 6.470 & AK054970 & 7.979 & HARIB & 6.887 & XLOC_013368 \\
\hline 5.052 & SNAR-A2 & 6.243 & HARIA & 6.325 & HARIA & 6.318 & NEATI \\
\hline 4.216 & FABP5P3 & 6.114 & XIST & 6.218 & SNAR-A2 & 6.205 & SEPT7P6 \\
\hline 4.082 & RP11-325F22.4 & 5.799 & MIAT & 6.066 & RP1 1-208G20.2 & 6.090 & CASC2 \\
\hline 3.887 & SEPT7P6 & 5.712 & SNAR-A2 & 5.652 & XLOC_008014 & 5.873 & TMSB10P2 \\
\hline
\end{tabular}

* = II+III - tumours of WHO grade II and III; (abs) = absolute value; FC = fold change

ray. We observed the highest average expression in oligoastrocytoma and oligodendroglioma, and statistical analysis of ANOVA showed significant differences comparing all groups $(\mathrm{p}=0.004)$. Cross comparison of two subtypes using Mann-Whitney test showed significantly increased expression between oligodendroglioma and astrocytoma I-III $(p=0.007)$, secondary GBM $(p=0.021)$, and primary GBM ( $p=0.014)$, respectively. Comparing astrocytoma to oligoastrocytoma showed lower expression and borderline value of significance $(\mathrm{p}=$ 0.051 ), and similar was observed in comparison of secondary GBM and oligoastrocytoma $(p=0.052)$ (Figure 2).

\section{Methylation status of LOC285758 promoter}

After we quantified and analysed the lncRNA expression, we wanted to see if this change could be a consequence of changed DNA methylation pattern. Methylation status of LOC285758 promoter was determined for individual sample from a subset of 125 samples with sufficient DNA concentration and quality. We compared sample's normalized and melting curve compared to fully methylated and fully unmethylated control. Fiftyone percent $(64 / 125)$ of samples were methylated and we found methylation status to be inversely associated with LOC285758 expression (Figure 3A) $\left(r_{s}=-0.455, p<0.001\right)$. We also compared normalized curves of tumour samples and control pools. LOC285758 promoter was shown as methylated in control pools (normal brain), but hypo-methylated against positive control, and $87 \%(109 / 125)$ of tumour samples were hypo-methylated or unmethylated in regard to normal brain. Further analysis, regarding the glioma subtype (Figure $3 \mathrm{C}$ ), showed astrocytoma of WHO grades I-III are methylated in $96 \%$ of samples, whereas astrocytoma of grade 
IV, i.e. GBM showed high percent of unmethylated cases (57\% of secondary GBM (12/21) and 77\% of primary GBM (20/26)). Cases of oligodendroglioma were unmethylated in $75 \%(21 / 28)$, whereas oligoastrocytoma were unmethylated in $44 \%(8 / 18)$ (Figure 3C). Also, classifying samples upon WHO grade showed tumours of lower grades are largely methylated (Figure 3B).

\section{Association of LOC285758 expression and methylation with glioma hallmark markers}

We wanted to search for a possible association of expression with already established glioma biomarkers. For determining mutation status of $I D H 1$ and IDH2 gene, variation in copy number of $C D K N 2 A$ and $C D K N 2 B$ gene, and deletion of chromosome arm $1 \mathrm{p}$ and $19 \mathrm{q}$ we conducted the MLPA analysis. Additionally, results of routine immunohistochemical analyses were collected from our database. TP53 was mutated in $38 \%$ of samples (59 mutations (MUT), 36 wild type (WT), and 62 not acquired (NA)). IDH1 mutation R132H was found in 34\% (54 MUT, $64 \mathrm{WT}$, and $38 \mathrm{NA}$ ) of samples, and $\mathrm{R} 132 \mathrm{C}$ in one case $(<1 \%)$. In $\mathrm{IDH} 2$ we did not find any mutations. $C D K N 2 A / C D K N 2 B$ were deleted in 23\% (37/37 deletions (DEL), 44/45 WT, 2/1 duplications (DUPL), and 74 NA). Chromosome arm $1 p$ was lost completely in 17\% (27 DEL, 7 partial DEL, 3 DUPL, 69 WT and 51 NA) and 19q in 19\% (30 DEL, 14 partial DEL, 11 DUPL, $49 \mathrm{WT}$, and $53 \mathrm{NA})$. Duplication of $19 \mathrm{q}$ was found mainly in GBM tumours. Co-deletion of $1 p$ and $19 q$ arm was detected in $15 \%$ of samples ( 24 cases) and majority of them were oligodendroglioma. For correlation analysis we excluded partial deletions and duplications of $C D K N 2 A / B, 1 \mathrm{p}$ and $19 \mathrm{q}$.

We compared both LOC285758 expression and promoter methylation status to above biomarkers. Pearson's correlation coefficient test showed both expression and methylation to be significantly associated with WHO malignancy grade (Figure 3B). Expression was also positively associated with the IDH1 mutation - samples with IDH1 mutation had higher expression, but independently from promoter's methylation. A more detailed analysis, regarding the subtype, showed expression of LOC285758 does not differ between IDH1 mutated and wildtype astrocytoma of lower grades. But significantly does in secondary GBM, oligoastrocytoma and oligodendroglioma. We found methylation to be in weak relation to age at diagnosis and loss of $C D K N 2 A$ and $C D K N 2 B$, but in an inverse

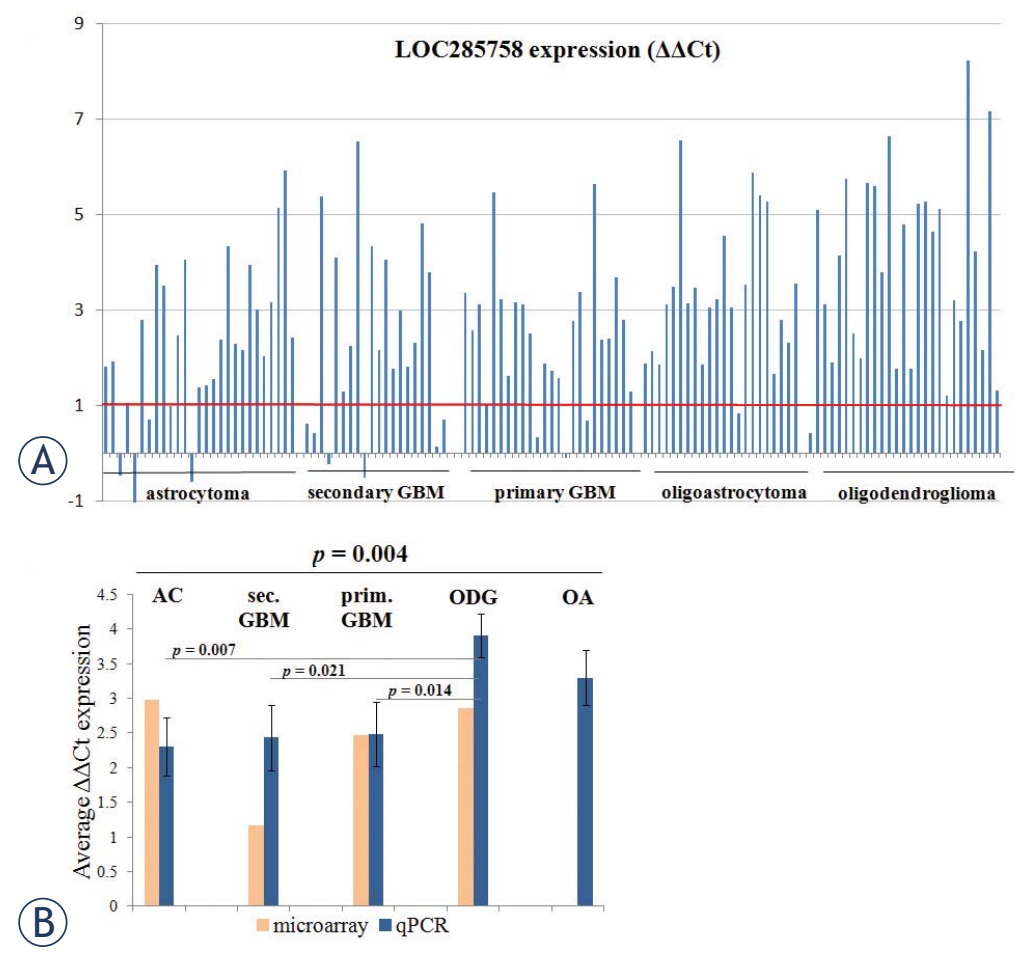

FIGURE 2. (A) Differential expression of LOC285758 in individual samples (y-axis presents $\Delta \Delta C_{T}$ values). (B) Comparison of average $\Delta \Delta C_{T}$ values for individual glioma subtype, determined by microarray and qPCR. Oligoastrocytoma samples were not included in microarray analysis. $\Delta \Delta \mathrm{C}_{\mathrm{T}}$ represents difference of gene's expression in comparison to brain reference RNA, and the positive values mean that gene's levels are increased. $p$-values were determined for QPCR data (ANOVA for comparing all five subtypes and Mann-Whitney U-test for comparing two subtypes).

TABLE 4. Association of LOC285758 expression with patients demographic data and glioma hallmark biomarkers: mutations of IDHI and TP53, copy number variations of CDKN2A and CDKN2B, and loss of chromosome arm $1 p$ and $19 q$ (1p/19q co-deletion)

\begin{tabular}{lcccc}
\hline & \multicolumn{2}{c}{ LOC285758 expression } & \multicolumn{2}{c}{$\begin{array}{c}\text { LOC285758 promoter } \\
\text { methylation }\end{array}$} \\
\cline { 2 - 5 } & $\mathrm{r}_{\mathrm{s}}$ & $\mathrm{p}$-value & $\mathrm{r}_{\mathrm{s}}$ & $\mathrm{p}$-value \\
\hline Gender & -0.044 & 0.634 & 0.009 & 0.920 \\
Age at diagnosis (<45y $>$ ) & 0.065 & 0.475 & -0.313 & $<0.001$ \\
WHO grade (low/high) & 0.213 & 0.019 & -0.433 & $<0.001$ \\
IDH1 (wt/mut) & 0.375 & $<0.001$ & 0.096 & 0.331 \\
TP53(wt/mut) & -0.083 & 0.483 & 0.153 & 0.178 \\
1p loss (wt/del) & 0.310 & 0.005 & -0.396 & $<0.001$ \\
19q loss (wt/del) & 0.267 & 0.032 & -0.360 & 0.002 \\
1p/19q loss (wt/del) & 0.262 & 0.014 & -0.373 & $<0.001$ \\
CDKN2A (wt/del) & 0.085 & 0.477 & -0.231 & 0.042 \\
CDKN2B (wt/del) & 0.093 & 0.435 & -0.240 & 0.033 \\
\hline
\end{tabular}

$r_{s}=$ Pearson's correlation/association coefficient (0.2-0.4- weak, 0.4-05-moderate, > 0.6 strong correlation); $p$-value cut-off is set at 0.05 ( $95 \%$ confidence interval) 

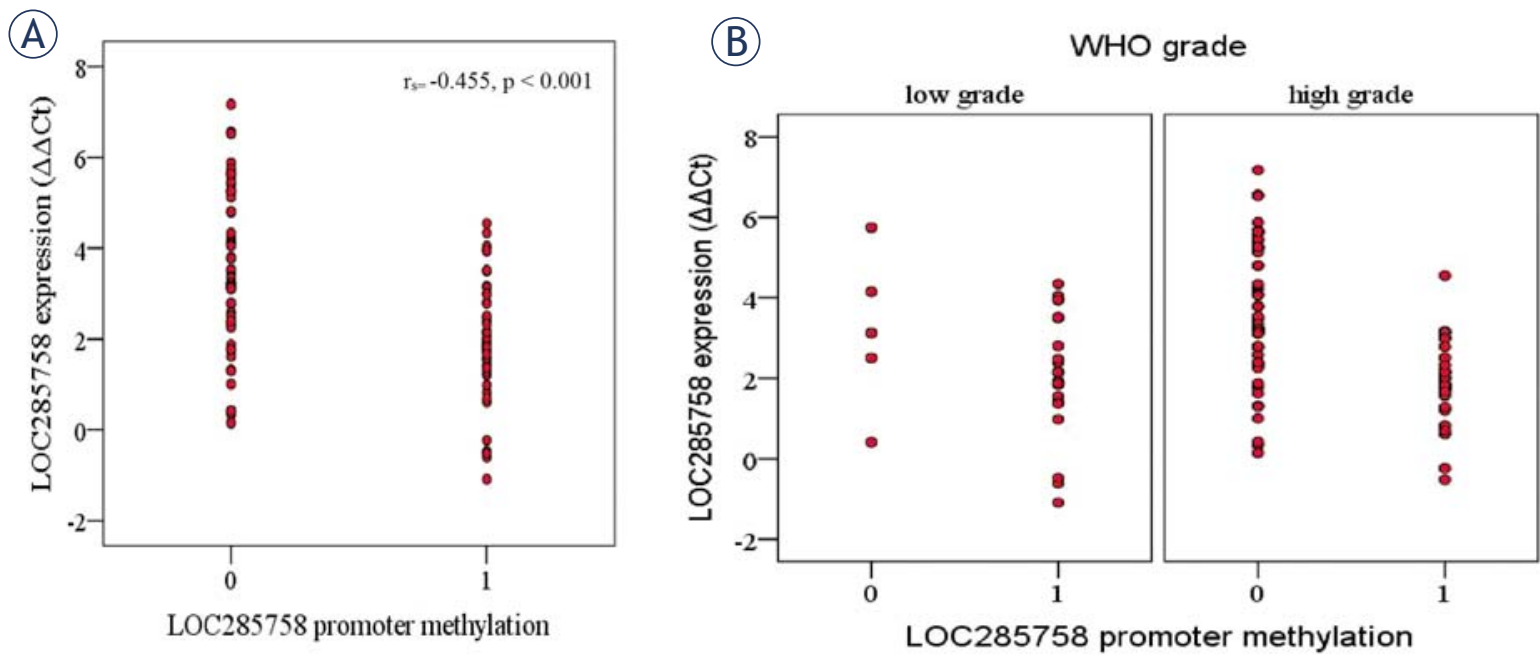

$\Delta \Delta \mathrm{C}_{\mathrm{T}}-\mathrm{WHO}$ grade $\mathrm{r}_{\mathrm{s}}=0.213, \mathrm{p}=0.019$; methylation - WHO grade $\mathrm{r}_{\mathrm{s}}=-0.433, \mathrm{p}<0.001$

(C)
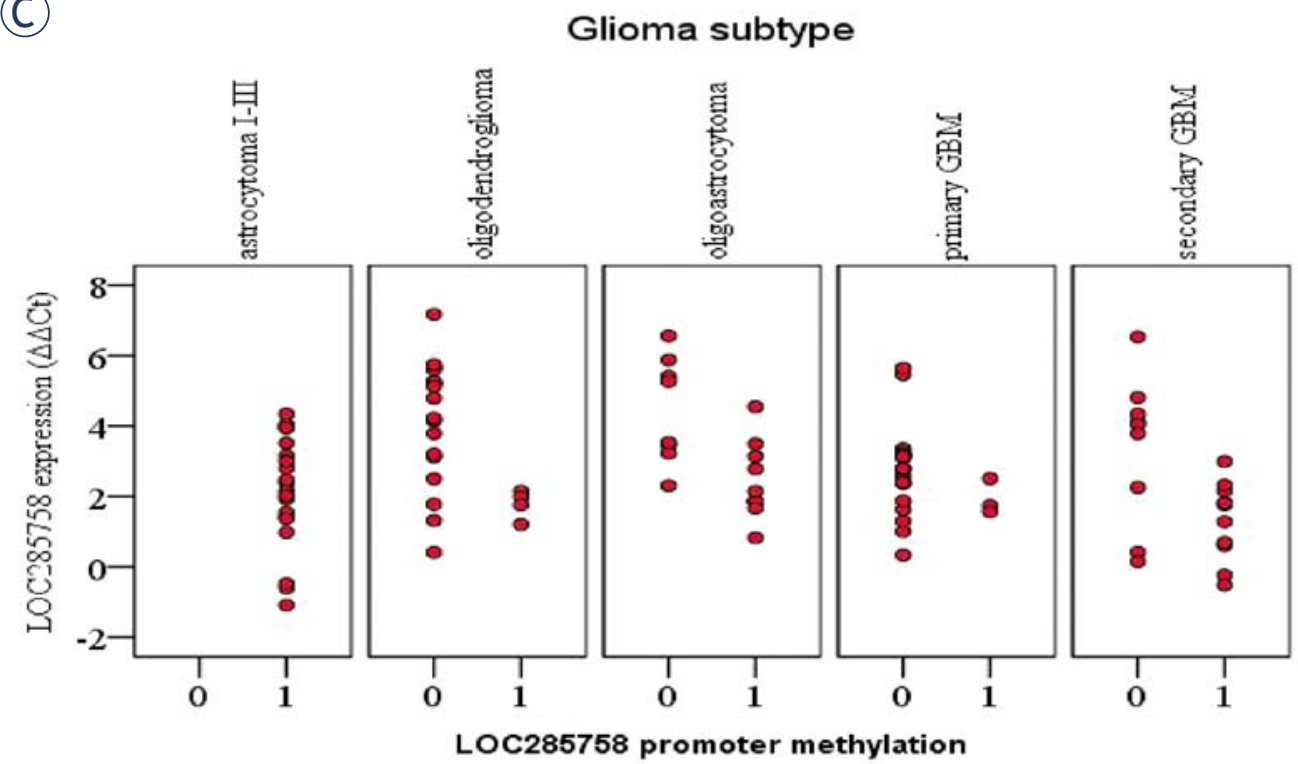

FIGURE 3. Scatter plots showing (A) LOC285758 expression (QPCR) in association to methylation status. Unmethylated samples showed higher expression levels compared to methylated ones. (B) LOC285758 expression and promoter methylation status significantly differ regarding the WHO malignancy grade and (C) glioma subtype, especially comparing astrocytoma grade I-III (all samples were methylated) to grade IV (GBMs were largely unmethylated).

Promoter methylation: 0 = unmethylated, $1=$ methylated

manner. The loss of chromosome arm $1 \mathrm{p}, 19 \mathrm{q}$ and both arms concurrently was associated with both expression and methylation (Table 4); however, as mentioned above, co-deletion was found mainly in oligodendroglioma.

\section{Discussion}

Due to the aggressive nature of glioma, poor prognosis and especially the resistance of tumour cells against established and/or even modern treatments there is a need for more precise definition of glioma molecular background and finding new biomarkers. The simplest way to screen through high number of genes potentially involved in tumour development is expression profiling using microarray technology. We analysed the differences in expression of lncRNAs, potentially involved in epigenetic regulatory mechanisms. Among 879 lncRNA microarray probes we identified 351 lncRNAs with significantly changed expression (with absolute 
fold change cut-off of 1.5) in at least one glioma subtype, which once again confirms molecularly heterogeneous nature of glioma tumours. ${ }^{1}$ We determined 60 lncRNAs with changed expression in all four analyzed subtypes and selected few lncRNAs due to their significantly changed expression either in or between all four subtypes either regarding a specific subtype. For many lncRNAs we did not find any previous reports; however, expression of few IncRNAs, such as MEG327,28, H1929, Xist ${ }^{30}$, and just recently $N E A T 1^{31}$ and $H O X A 11-A S^{32}$, was already shown to be significantly changed in glioma. Moreover, they were associated with disturbance of key cellular pathways and patient's prognosis.

To the best of our knowledge, one of the unknown cancer-related lncRNAs is LOC285758, or long intergenic non-protein coding RNA 1268 (LINC01268), that lies in close proximity of the MARCKS (myristoylated alanine-rich C-kinase substrate) gene, which encodes a cell cycle and motility promoting protein. As LOC285758 expression on microarray appeared to be significantly lower in secondary GBM in comparison to primary GBM it represented a candidate biomarker for potentially distinguish these two entities, especially since many cases are morphologically alike and thus misclassified. ${ }^{6}$ However, validation results showed expression does not significantly differ between astrocytic tumours, but methylation status does as almost all primary GBM were un-methylated versus variable status in secondary GBM. This variation in DNA methylation might be associated with secondary GBM developing through low-grade astrocytic precursors ${ }^{33}$, since we found almost all astrocytic tumours of lower grades methylated. It could also indicate that methylation pattern of lncRNA's promoter changes as the tumour progresses.

Nevertheless, LOC285758 expression levels do significantly differ between astrocytoma, oligoastrocytoma and oligodendroglioma, respectively, which could also be a helpful discriminating feature of oligoastrocytoma from astrocytic tumours of grade I-III, since these neoplasms are somewhat a mix of genetic changes characteristic for astrocytic (IDH1 mutations) and oligodendroglial tumours (1p/19q co-deletion). ${ }^{1}$ Expression levels and methylation status of LOC285758 found in oligoastrocytoma, compared to both subtypes, even more corroborate their mixed genetic background. Additionally, LOC285758 could be an additional feature to distinguish oligodendroglioma and astrocytoma, since these entities might arise from the same cells of origin, but develop through different molecular pathways. ${ }^{1}$
Results of different methylation further highlight epigenetic alterations of lncRNAs. We chose the MS-HRM method for determining methylation status of gene's promoter as it is simple, fast and highly sensitive; even one single base substitution can be observed as a significant fluorescence drop and melting peak shift. ${ }^{34,35}$

As lncRNAs principal mechanism is regulation of coding genes and many bind to chromatin-modifying genes ${ }^{17}$, the potential targets of LOC285758 could be addressed by its genome location, with MARCKS and HDAC2 as neighbouring genes. In contrast to the anonymity of LOC585758, there are numerous reports of MARCKS being involved in various cancers, including glioma. ${ }^{36-39}$ It has the ability to regulate various signalling pathways; by sequestering the phosphatidylinositol 4,5-biphosphate (PIP2) molecules, a PI3K target, MARCKS functions as a tumour suppressor of PI3K/Akt signalling pathway. ${ }^{40,41}$ On contrary, it can also function as tumour enhancer as its overexpression was associated with metastasis and poorer prognosis in patients with lung squamous cell carcinoma ${ }^{42}$, and promoting EGFRvIII-mediated GBM tumorigenesis. ${ }^{39}$ There is only one report showing association of LOC285758 expression with expression of its antisense mRNA, and changed methylation of intragenic $\mathrm{CpG}$ island; however, it was not cancer related. ${ }^{43} H D A C 2$ is an enzyme of histone deacetylase family, the key players in epigenetic silencing of gene expression and one of the regulators of major cellular functions, such as cell cycle, apoptosis, DNA damage repair, and senescence. ${ }^{44,45}$ Like MARCKS, HDAC2 is already extensively studied, largely due to its crucial biological function, and was found significantly deregulated in broad spectrum of diseases. ${ }^{46-50} \mathrm{HDAC} 2$ was overexpressed in astrocytoma, like $H D A C 1$, and expression increases during tumour recurrence and progression, but it is not WHO-grade-related. ${ }^{50}$ Whether the functional association of LOC285758 and its neighbouring genes exist is yet to be investigated.

Some limitations of our study are related to collecting patient's data as no complete clinical data were available at the time of the study. Another limitation is the lack of knowledge about exact functions and roles of lncRNA LOC285758 in normal brain and cancer tissue, but such studies are beyond our research timeframe.

We can conclude the microarray expression profiling of glioma tumours proved useful for identification of novel lncRNAs involved in epigenetic pathways of glioma development as we determined several potential lncRNAs with sig- 
nificantly changed expression, including lncRNA LOC285758. Our study is, to the best of our knowledge, the first to report disturbed expression of LOC285758 in glioma tumours. Elevated expression of LOC285758 and its association to loss of cytosine methylation and higher malignancy grade suggest to an oncogenic function of this lncRNA in glioma biogenesis. Furthermore, significantly different expression between astrocytoma and oligodendroglioma, and oligoastrocytoma, respectively, might suggests LOC285758 as a new biomarker candidate of glioma development with added diagnostic value, but its exact function is yet to be revealed.

\section{Acknowledgment}

This research is a part of Alenka Matjašič doctoral thesis. This study was supported by the Slovenian Research Agency (P3-054).

\section{References}

1. Louis DN, Molecular pathology of malignant gliomas. Annu Rev Pathol 2006; 1: 97-117. doi:10.1146/annurev.pathol.1.110304.100043

2. Mesti T, Moltara ME, Boc M, Rebersek M,Ocvirk J, Bevacizumab and irinotecan in recurrent malignant glioma, a single institution experience. Radiol Oncol 2015; 49: 80-5. doi:10.2478/raon-2014-0021

3. Qureshi IA, Mattick JS,Mehler MF, Long non-coding RNAs in nervous system function and disease. Brain Res 2010; 1338: 20-35. doi:10.1016/j.brainres.2010.03.110

4. Gibb EA, Vucic EA, Enfield KS, Stewart GL, Lonergan KM, Kennett JY, et al., Human cancer long non-coding RNA transcriptomes. PLoS One 2011; 6: e25915. doi:10.1371/journal.pone.0025915

5. Qureshi IA,Mehler MF, Emerging roles of non-coding RNAs in brain evolution, development, plasticity and disease. Nat Rev Neurosci 2012; 13: 528 41. doi:10.1038/nrn3234

6. Zhang X, Sun S, Pu JK, Tsang AC, Lee D, Man VO, et al., Long non-coding RNA expression profiles predict clinical phenotypes in glioma. Neurobiol Dis 2012; 48: 1-8. doi:10.1016/j.nbd.2012.06.004

7. Gibb EA, Brown CJ,Lam WL, The functional role of long non-coding RNA in human carcinomas. Mol Cancer 2011; 10: 38. doi:10.1186/1476-4598-10-

8. Guttman M, Amit I, Garber M, French C, Lin MF, Feldser D, et al., Chromatin signature reveals over a thousand highly conserved large non-coding RNAs in mammals. Nature 2009; 458: 223-7. doi:10.1038/nature07672

9. Djebali S, Davis CA, Merkel A, Dobin A, Lassmann T, Mortazavi A, et al., Landscape of transcription in human cells. Nature 2012; 489: 101-8. doi:10.1038/nature11233

10. Weichenhan D,Plass C, The evolving epigenome. Hum Mol Genet 2013; 22 R1-6. doi:10.1093/hmg/ddt348

11. Hassler MR,Egger G, Epigenomics of cancer - emerging new concepts Biochimie 2012; 94: 2219-30. doi:10.1016/j.biochi.2012.05.007

12. Tripathi V, Shen Z, Chakraborty A, Giri S, Freier SM, Wu X, et al., Long noncoding RNA MALAT1 controls cell cycle progression by regulating the expression of oncogenic transcription factor B-MYB. PLoS Genet 2013; 9: e1003368. doi:10.1371/journal.pgen.1003368
13. Tian $D$, Sun S,Lee JT, The long noncoding RNA, Jpx, is a molecular switch for X chromosome inactivation. Cell 2010; 143: 390-403. doi:10.1016/j. cell.2010.09.049

14. Lee $\mathrm{C}$,Kikyo $\mathrm{N}$, Strategies to identify long noncoding RNAs involved in gene regulation. Cell Biosci 2012; 2: 37. doi:10.1186/2045-3701-2-37.

15. Kitagawa M, Kitagawa $\mathrm{K}$, Kotake $\mathrm{Y}$, Niida $\mathrm{H}, \mathrm{Ohhata} \mathrm{T}$, Cell cycle regulation by long non-coding RNAs. Cell Mol Life Sci 2013; 70: 4785-94. doi:10.1007/ s00018-013-1423-0

16. Han L, Zhang K, Shi Z, Zhang J, Zhu J, Zhu S, et al., LncRNA pro fi le of glioblastoma reveals the potential role of IncRNAs in contributing to glioblastoma pathogenesis. Int J Oncol 2012; 40: 2004-12. doi:10.3892/ijo.2012.1413

17. Mercer TR,Mattick JS, Structure and function of long noncoding RNAs in epigenetic regulation. Nat Struct Mol Biol 2013; 20: 300-7. doi:10.1038/ nsmb. 2480

18. Karapetyan AR, Buiting C, Kuiper RA, Coolen MW, Regulatory roles for Long ncRNA and mRNA. Cancers (Basel) 2013; 5: 462-90. doi:10.3390/ cancers5020462

19. Kanduri C, Long noncoding RNAs: Lessons from genomic imprinting. Biochim Biophys Acta 2016; 1859: 102-11. doi:10.1016/j.bbagrm.2015.05.006

20. Fietkau R, Putz F, Lahmer G, Semrau S,Buslei R, Can MGMT promoter methylation status be used as a prognostic and predictive marker for glioblastoma multiforme at the present time? A word of caution. Strahlenther Onkol 2013; 189: 993-5. doi:10.1007/s00066-013-0459-2

21. Smrdel U, Popovic M, Zwitter M, Bostjancic E, Zupan A, Kovac V, et al., Longterm survival in glioblastoma: methyl guanine methyl transferase (MGMT) promoter methylation as independent favourable prognostic factor. Radiol Oncol 2016; 50: 394-401. doi:10.1515/raon-2015-0041

22. Shi $X$, Sun $M$, Liu $H$, Yao $Y$,Song $Y$, Long non-coding RNAs: a new frontier in the study of human diseases. Cancer Lett 2013; 339: 159-66. doi:10.1016/j. canlet.2013.06.013

23. Bian EB, Li J, Xie YS, Zong G, Zhao B, LncRNAs: New players in gliomas, with special emphasis on the interaction of IncRNAs with EZH2. J Cell Physio 2015; 230: 496-503. doi:10.1002/jcp.24549

24. Alelu-Paz R, Ashour N, Gonzalez-Corpas A,Ropero S, DNA methylation, histone modifications, and signal transduction pathways: a close relationship in malignant gliomas pathophysiology. J Signal Transduct 2012; 2012: 956958. doi:10.1155/2012/956958

25. Louis DN, Ohgaki H, Wiestler OD, Cavenee WK, Burger PC, Jouvet A, et al., The 2007 WHO classification of tumours of the central nervous system. Acto Neuropathol 2007; 114: 97-109. doi:10.1007/s00401-007-0243-4

26. Livak KJ,Schmittgen TD, Analysis of relative gene expression data using realtime quantitative PCR and the 2(-Delta Delta C(T)) Method. Methods 2001; 25: 402-8. doi:10.1006/meth.2001.1262

27. لـ إن Bian EB, He XJ, Ma CC, Zong G, Wang HL, et al., Epigenetic repression of long non-coding RNA MEG3 mediated by DNMT1 represses the p53 pathway in gliomas. Int J Oncol 2016; 48: 723-33. doi:10.3892/ijo.2015.3285

28. Wang $P$, Ren Z,Sun $P$, Overexpression of the long non-coding RNA MEG3 impairs in vitro glioma cell proliferation. J Cell Biochem 2012; 113: 1868-74. doi:10.1002/jcb.24055

29. Park JY, Lee JE, Park JB, Yoo H, Lee SH, Kim JH, Roles of long non-Coding RNAs on tumorigenesis and glioma development. Brain Tumor Res Treat 2014; 2 1-6. doi:10.14791/btrt.2014.2.1.1

30. Yao Y, Ma J, Xue Y, Wang P, Li Z, Liu J, et al., Knockdown of long non-coding RNA XIST exerts tumor-suppressive functions in human glioblastoma stem cells by up-regulating miR-152. Cancer Lett 2015; 359: 75-86. doi:10.1016/j. canlet.2014.12.051

31. He C, Jiang B, Ma J,Li Q, Aberrant NEAT1 expression is associated with clinical outcome in high grade glioma patients. Apmis 2016; 124: 169-74. doi:10.1111/apm.12480

32. Wang Q, Zhang J, Liu Y, Zhang W, Zhou J, Duan R, et al., A novel cell cycleassociated IncRNA, HOXA11-AS, is transcribed from the 5-prime end of the HOXA transcript and is a biomarker of progression in glioma. Cancer Lett 2016; 373: 251-9. doi:10.1016/j.canlet.2016.01.039

33. Ohgaki $H$,Kleihues $P$, The definition of primary and secondary glioblastoma. Clin Cancer Res 2013; 19: 764-72. doi:10.1158/1078-0432.ccr-12-3002 
34. Wojdacz TK,Dobrovic A, Methylation-sensitive high resolution melting (MSHRM): a new approach for sensitive and high-throughput assessment of methylation. Nucleic Acids Res 2007; 35: e41. doi:10.1093/nar/gkm013

35. Switzeny OJ, Christmann M, Renovanz M, Giese A, Sommer C,Kaina B, MGMT promoter methylation determined by HRM in comparison to MSP and pyrosequencing for predicting high-grade glioma response. Clin Epigenetics 2016; 8: 49. doi:10.1186/s13148-016-0204-7

36. Hagedorn M, Siegfried G, Hooks KB,Khatib AM, Integration of zebrafish fin regeneration genes with expression data of human tumors in silico uncovers potential novel melanoma markers. Oncotarget 2016; 7: 71567-79. doi:10.18632/oncotarget.12257

37. Legendre CR, Demeure MJ, Whitsett TG, Gooden GC, Bussey KJ, Jung S, et al., Pathway implications of aberrant global methylation in adrenocortical cancer. PLoS One 2016; 11: e0150629. doi:10.1371/journal.pone.0150629

38. Yang Z, X U S, Jin P, Yang X, Li X, Wan D, et al., MARCKS contributes to stromal cancer-associated fibroblast activation and facilitates ovarian cancer metastasis. Oncotarget 2016; 7: 37649-63. doi:10.18632/oncotarget.8726

39. Micallef J, Taccone M, Mukherjee J, Croul S, Busby J, Moran MF, et al., Epidermal growth factor receptor variant III-induced glioma invasion is mediated through myristoylated alanine-rich protein kinase $C$ substrate overexpression. Cancer Res 2009; 69: 7548-56. doi:10.1158/0008-5472. can-08-4783

40. Rohrbach TD, Shah N, Jackson WP, Feeney EV, Scanlon S, Gish R, et al., The effector domain of MARCKS is a nuclear localization signal that regulates cellular PIP2 levels and nuclear PIP2 localization. PLoS One 2015; 10: e0140870. doi:10.1371/journal.pone.0140870

41. Rohrbach TD, Jarboe JS, Anderson JC, Trummell HQ, Hicks PH, Weaver AN, et al., Targeting the effector domain of the myristoylated alanine rich C-kinase substrate enhances lung cancer radiation sensitivity. Int J Oncol 2015; 46: 1079-88. doi:10.3892/ijo.2014.2799

42. Hanada S, Kakehashi A, Nishiyama N, Wei M, Yamano S, Chung K, et al., Myristoylated alanine-rich C-kinase substrate as a prognostic biomarker in human primary lung squamous cell carcinoma. Cancer Biomark 2013; 13 : 289-98. doi:10.3233/cbm-130354

43. Punzi G, Ursini G, Shin JH, Kleinman JE, Hyde TM,Weinberger DR, Increased expression of MARCKS in post-mortem brain of violent suicide completers is related to transcription of a long, noncoding, antisense RNA. Mol Psychiatry 2014; 19: 1057-9. doi:10.1038/mp.2014.41

44. Ropero S,Esteller M, The role of histone deacetylases (HDACS) in human cancer. Mol Oncol 2007; 1: 19-25. doi:10.1016/j.molonc.2007.01.001

45. Li Z,Zhu WG, Targeting histone deacetylases for cancer therapy: from molecular mechanisms to clinical implications. Int J Biol Sci 2014; 10: 757-70. doi:10.7150/ijbs.9067

46. Conte M, Dell'Aversana C, Benedetti R, Petraglia F, Carissimo A, Petrizzi $\mathrm{VB}$, et al., HDAC2 deregulation in tumorigenesis is causally connected to repression of immune modulation and defense escape. Oncotarget 2015; 6: 886-901. doi:10.18632/oncotarget.2816

47. Alzoubi S, Brody L, Rahman S, Mahul-Mellier AL, Mercado N, Ito K, et al, Synergy between histone deacetylase inhibitors and DNA-damaging agents is mediated by histone deacetylase 2 in colorectal cancer. Oncotarget 2016; 7: 44505-21. doi:10.18632/oncotarget.9887

48. Qu X, Yu H, Jia B, Yu X, Cui Q, Liu Z, et al., Association of downregulated HDAC 2 with the impaired mitochondrial function and cytokine secretion in the monocytes/macrophages from gestational diabetes mellitus patients. Cell Biol Int 2016; 40: 642-51. doi:10.1002/cbin.10598

49. Stojanovic N, Hassan Z, Wirth M, Wenzel P, Beyer M, Schafer C, et al., HDAC1 and HDAC2 integrate the expression of p53 mutants in pancreatic cancer. Oncogene 2016. doi:10.1038/onc.2016.344

50. Campos B, Bermejo JL, Han L, Felsberg J, Ahmadi R, Grabe N, et al., Expression of nuclear receptor corepressors and class I histone deacetylases in astrocytic gliomas. Cancer Sci 2011; 102: 387-92. doi:10.1111/j.1349 7006.2010.01792 